\title{
The association of neutrophil to lymphocyte ratio with presence and severity of obstructive sleep apnea
}

\author{
Sunbul $\mathrm{M}^{1}$, Sunbul EA ${ }^{2}$, Kanar B ${ }^{1}$, Yanartas $\mathrm{O}^{3}$, Aydin $\mathrm{S}^{4}$, Bacak A $\mathrm{A}^{5}$, Gulec $\mathrm{H}^{2}$, Sari $\mathrm{I}^{1}$ \\ Marmara University Faculty of Medicine, Department of Cardiology, Istanbul, Turkey. \\ drsunbul@yahoo.com.tr
}

\begin{abstract}
BACKGROUND: Obstructive sleep apnea (OSA) is characterized by reductions in breathing amplitude during sleep caused by an obstructed or collapsed upper airway. The neutrophil to lymphocyte ratio (NLR) has been proposed as a novel biomarker for systemic inflammatory response. The aim of the present study was to evaluate the relationship between NLR and OSA.

METHODS: The study population consisted of 195 consecutive patients with clinical suspicion of OSA. Full night polysomnography was performed for all patients. Patients with an apnea hypopnea index (AHI) $\geq 5$ were considered to have OSA. NLR was calculated as the ratio of neutrophil count to lymphocyte count.

RESULTS: While 130 patients (91 male, mean age: $49.9 \pm 9.8$ years) had OSA, 65 patients (42 male, mean age: $48.7 \pm 10.2$ years) had normal findings according to AHI scores. Cardiovascular risk factors such as hypertension, hyperlipidemia, diabetes mellitus and smoking were more common in patients with OSA. Patients with OSA had significantly higher NLR than controls $(1.87 \pm 0.80$ vs $1.49 \pm 0.48, p=0.005)$. AHI score was significantly correlated with NLR $(r=0.228, p=0.001)$. NLR and presence of hyperlipidemia were independent predictors of OSA (OR: $2.451,2.850, p=0.001,0.004$, respectively). NLR of 1.62 or higher predicted OSA with a sensitivity of $56.2 \%$ and specificity of $63.1 \%$.

CONCLUSIONS: NLR was higher and also correlated with AHI score in patients with OSA which has not been reported previously. NLR more than 1.62 was an independent predictor of OSA. A simple, cheap white blood cell count may also give an idea about the presence and severity of OSA (Tab. 3, Fig. 3, Ref. 30). Text in PDF www.elis.sk. KEY WORDS: inflammation, cardiovascular risk, neutrophil to lymphocyte ratio, obstructive sleep apnea.
\end{abstract}

\section{Introduction}

Obstructive sleep apnea (OSA) is characterized by reductions in breathing amplitude during sleep caused by complete or partial obstruction of the upper airways (1). OSA usually results in significant arterial hypoxemia and hypercapnia. The incidence of OSA is approximately $4 \%$ in adult population (2). OSA is an important health problem that is associated with several diseases including hypertension (HT), obesity, diabetes mellitus (DM), and cardiovascular disease (CVD) (3). Several factors have been proposed for the relationship between OSA and cardiovascular risk factors. Inflammation is the major factor in the development and progression of CVD (4). OSA is associated with pro-inflammatory and pro-thrombotic mediators (5-7). Inflammatory mediators con-

${ }^{1}$ Marmara University Faculty of Medicine, Department of Cardiology, Istanbul, Turkey, ${ }^{2}$ Erenkoy Training and Research Hospital for Psychiatric and Neurological Disorders, Psychiatry Clinic, Istanbul, Turkey, ${ }^{3}$ Marmara University Faculty of Medicine, Department of Psychiatry, Istanbul, Turkey, ${ }^{4}$ Yedikule Chest Disease and Thorax Surgery Education and Research Hospital, Department of Neurology, Istanbul Turkey, and ${ }^{5}$ Yedikule Chest Disease and Thorax Surgery Education and Research Hospital, Sleep Laboratory, Istanbul Turkey

Address for correspondence: M. Sunbul, MD, Fevzi Cakmak Mah. Muhsin Yazicioglu Cad. No: 10, Üst Kaynarca, Pendik, Istanbul, Turkey.

Phone: +90.506 .5819015 , Fax: +90.216 .6570695$ tribute to the development of atherosclerosis in patients with OSA. Previous studies have demonstrated that OSA is an independent risk factor for development of $\operatorname{CVD}(8,9)$.

White blood cell count and its components such as neutrophils and lymphocytes have important role in inflammatory processes. The neutrophil to lymphocyte ratio (NLR) which can be derived from the white blood cell count is an inexpensive, routinely used, reproducible test. NLR has been proposed as a novel biomarker for systemic inflammatory response in several diseases (10-13). Previous studies have shown that NLR is associated with poor clinical outcomes in CVD and it is also a predictor for CVD (10, 12). While the association between NLR and CVD is well known, the association between NLR and OSA remains unclear. The aim of the present study was to evaluate the relationship between NLR and OSA which has not been studied previously.

\section{Materials and methods}

Study population

Participants in this study were recruited from the patient population of the Yedikule Chest Disease and Thorax Surgery Education and Research Hospital, Istanbul. 195 consecutive patients between ages of 20 and 75 who were admitted to sleep disorders center with a clinical suspicion of OSA were enrolled into the study. All participants underwent a standard polysomnography (overnight) by 
Tab. 1. Baseline characteristics and clinical data of the study population.

\begin{tabular}{|c|c|c|c|c|c|}
\hline & $\begin{array}{l}\text { Mild OSA } \\
(n=20)\end{array}$ & $\begin{array}{l}\text { Moderate OSA } \\
\quad(n=35)\end{array}$ & $\begin{array}{l}\text { Severe OSA } \\
(n=75)\end{array}$ & $\begin{array}{l}\text { Controls } \\
(\mathrm{n}=65)\end{array}$ & $\mathrm{p}$ \\
\hline Age, (years) & $47.4 \pm 8.5$ & $50.8 \pm 9.6$ & $50.1 \pm 10.3$ & $48.7 \pm 10.2$ & 0.379 \\
\hline Male Gender, n & 12 & 26 & 53 & 42 & 0.612 \\
\hline BMI, kg/m² & $33.4 \pm 8.8$ & $33.1 \pm 5.1$ & $35.3 \pm 8.5$ & $26.9 \pm 4.9$ & $<0.001^{*,+, \alpha}$ \\
\hline $\mathrm{HR}$, beat/minute & $69.8 \pm 16.9$ & $65.6 \pm 13.0$ & $67.8 \pm 11.9$ & $60.3 \pm 10.3$ & $<0.003^{*,+, \alpha}$ \\
\hline $\mathrm{HT}, \mathrm{n}$ & 5 & 10 & 19 & 10 & 0.386 \\
\hline DM, n & 2 & 6 & 11 & 3 & 0.173 \\
\hline HL, $n$ & 15 & 20 & 52 & 29 & $0.011^{*,+, \alpha}$ \\
\hline Smoking, $\mathrm{n}$ & 4 & 7 & 16 & 9 & 0.702 \\
\hline
\end{tabular}

Data are presented as mean \pm standard deviation or number of patient.

OSA - Obstructive sleep apnea, BMI - Body mass index, HR - Heart Rate, HT - hypertension, DM - Diabetes mellitus, HL - Hyperlipidemia

Post-hoc analysis: " $\mathrm{p}<0.05$ between patients with mild OSA and controls, ${ }^{+} \mathrm{p}<0.05$ between patients with moderate OSA and controls, ${ }^{\alpha} \mathrm{p}<0.05$ between patients with severe OSA and controls

using the Embla A-10 data acquisition and analysis system (Embla; Medcare Flaga; Reykjavik, Iceland) in a sleep laboratory. Patients with an apnea hypopnea index (AHI) scores $\geq 5$ were considered to have OSA. Patients with AHI scores $<5$ were considered the control group. Patients with OSA were divided into four groups according to AHI scores - normal, mild OSA, moderate OSA, and severe OSA $(<5,5-15,16-30,>30$, respectively). Patients with systemic disease and with medical treatment to affect the white blood cell counts, such as hematopoietic system disorders, history of malignancies and/or treatment with chemotherapy, evidence of any concomitant inflammatory disease, acute infection, and chronic inflammatory status, acute coronary syndrome (ACS), history of glucocorticoid therapy within the past 3 months, secondary HT, heart failure, history of chronic renal or hepatic disease and cerebrovascular disease were excluded from the study.

Laboratory parameters were recorded from patient medical charts. NLR was calculated as the ratio of neutrophil count to lymphocyte count. The study population was evaluated for presence of cardiovascular risk factors such as hypertension (HT), hyperlipidemia (HL), and diabetes mellitus (DM). HT was defined as systolic blood pressure $\geq 140 \mathrm{~mm} \mathrm{Hg}$ and/or diastolic blood pressure $\geq 90$ $\mathrm{mm} \mathrm{Hg}$, previously diagnosed HT, or use of any antihypertensive medications. DM was defined as fasting plasma glucose levels more than $126 \mathrm{mg} / \mathrm{dL}$ in multiple measurements, previously diagnosed DM or use of antidiabetic medications such as oral antidiabetic agents or insulin. HL was defined as serum total cholesterol $\geq 240 \mathrm{mg} / \mathrm{dl}$, serum triglyceride $\geq 200 \mathrm{mg} / \mathrm{dl}$, low-density lipoprotein cholesterol (LDL) $\geq 130 \mathrm{mg} / \mathrm{dl}$, previously diagnosed $\mathrm{HL}$, or use of lipid-lowering medication. Body mass index (BMI) was calculated by dividing the body weight in kilograms by the square of the height in meters. Smoking status was defined as the history of tobacco use at admission or in the 6 months prior to visit.

The investigation complies with the principles outlined in the Declaration of Helsinki. The study was approved by the local ethics committee and informed consent was provided from all participants.

\section{Statistical analysis}

Statistical analyses were performed using SPSS 20.0 statistical package for Windows (IBM SPSS, Armonk, NY, USA). Continuous data were expressed as mean \pm standard deviation while categorical data were presented as percentage. Normal distribution was assessed by Kolmogorov Smirnov test. Chi-square test was used for comparison of categorical variables while student T-test, Mann-Whitney U test or Kruskal Wallis tests were used to compare parametric and nonparametric continuous variables. Post-hoc analysis was performed by Bonferroni test. Correlation analysis was performed by Pearson or Spearman's correlation test. Logistic regression and linear regression analysis were performed to determine the predictors of OSA. Receiver operating characteristic (ROC) curve analysis was performed to determine the cut-off level of NLR to predict the presence of OSA. A value of $p<0.05$ was considered statistically significant.

\section{Results}

Baseline characteristics and clinical data are shown in Table 1. While 130 patients (91 male, mean age: $49.9 \pm 9.8$ years) had OSA, 65 patients (42 male, mean age: $48.7 \pm 10.2$ years) had normal findings ( $\mathrm{p}=0.447,0.539$, respectively) according to AHI scores. Mean AHI scores of patients with OSA and controls were $40.1 \pm$ 26.5 and $2.6 \pm 1.6$, respectively. Mean AHI scores were similar in both genders in patients with OSA $(40.3 \pm 25.8$ for male patients and $39.5 \pm 28.3$ for female patients, $\mathrm{p}=0.869$ ). Cardiovascular risk factors such as HT, HL, DM and smoking were more common in patients with OSA than controls although the difference

Tab. 2. Comparison of laboratory findings between patients and controls.

\begin{tabular}{lccc}
\hline & $\begin{array}{c}\text { Patient Group } \\
(\mathrm{n}=130)\end{array}$ & $\begin{array}{c}\text { Control Group } \\
(\mathrm{n}=65)\end{array}$ & $\mathrm{p}$ \\
\hline Leukocytes $\left(\mathrm{mm}^{-3}\right)$ & $8688 \pm 2186$ & $6936 \pm 1907$ & $<0.001$ \\
Neutrophils $\left(\mathrm{mm}^{-3}\right)$ & $4824 \pm 1648$ & $3596 \pm 1156$ & $<0.001$ \\
Lymphocytes $\left(\mathrm{mm}^{-3}\right)$ & $2835 \pm 967$ & $2623 \pm 1038$ & 0.052 \\
Platelets $\left(10^{3} / \mathrm{mm}^{-3}\right)$ & $269.9 \pm 74.6$ & $249.5 \pm 75.7$ & 0.075 \\
Hemoglobin $(\mathrm{g} / \mathrm{dL})$ & $14.9 \pm 1.6$ & $14.2 \pm 1.5$ & 0.005 \\
Fasting glucose $(\mathrm{mg} / \mathrm{dl})$ & $110.2 \pm 31.6$ & $100.9 \pm 21.7$ & 0.024 \\
Total cholesterol $(\mathrm{mg} / \mathrm{dl})$ & $218.7 \pm 45.1$ & $201.2 \pm 52.0$ & 0.004 \\
Triglycerides $(\mathrm{mg} / \mathrm{dl})$ & $183.6 \pm 101.6$ & $151.9 \pm 92.7$ & 0.017 \\
Low density lipoprotein $(\mathrm{mg} / \mathrm{dl})$ & $139.0 \pm 31.1$ & $118.2 \pm 36.9$ & $<0.001$ \\
High density lipoprotein $(\mathrm{mg} / \mathrm{dl})$ & $42.1 \pm 8.5$ & $45.4 \pm 9.5$ & 0.008 \\
C-reactive protein $(\mathrm{mg} / \mathrm{L})$ & $6.6 \pm 5.3$ & $2.5 \pm 2.4$ & $<0.001$ \\
Neutrophil to lymphocyte ratio & $1.87 \pm 0.80$ & $1.49 \pm 0.48$ & 0.005 \\
\hline
\end{tabular}

Data are expressed as mean $\pm \mathrm{SD}$ 


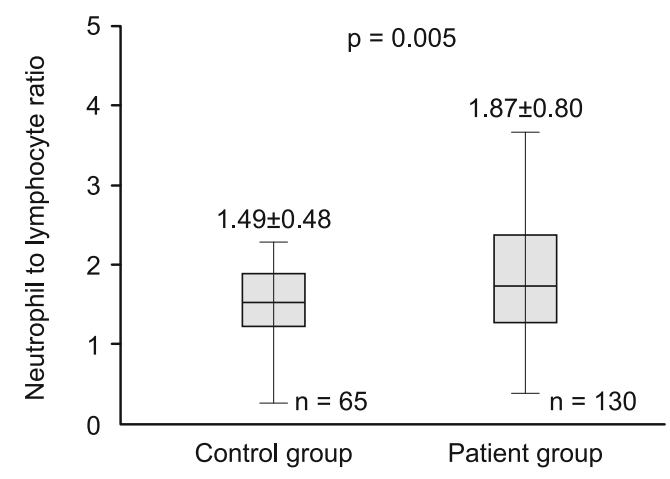

Fig. 1. Comparison of neutrophil to lymphocyte ratio between patients with obstructive sleep apnea and controls.

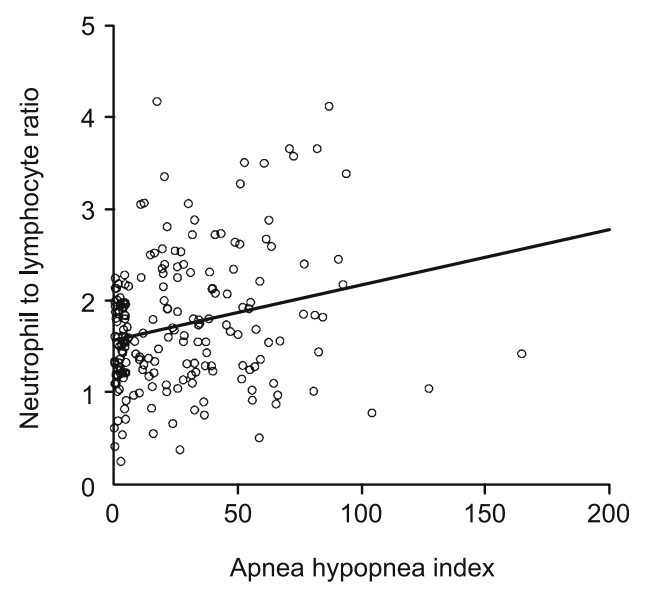

Fig. 2. Correlation analysis between neutrophil to lymphocyte ratio and apnea hypopnea index scores.

was non-significant. BMI and heart rate were significantly higher in patients with OSA than controls.

Laboratory findings are shown in Table 2. Fasting glucose, total cholesterol, triglyceride and LDL levels were significantly higher and high density lipoprotein (HDL) level was significantly lower in patients with OSA. Patients with OSA had significantly higher NLR than controls $(1.87 \pm 0.80$ vs $1.49 \pm 0.48, \mathrm{p}=0.005)$ (Fig. 1). C-reactive protein (CRP) level was also significantly higher in patients with OSA. Patients were divided into four groups according to AHI score such as normal, mild, moderate, and severe $(<5$,

Tab. 3. Multivariate analysis to determin the independent predictors of presence of obstructive sleep apnea.

\begin{tabular}{lccc}
\hline & Odds Ratio & $\begin{array}{c}95 \% \text { Confidence } \\
\text { Interval }\end{array}$ & $\mathrm{p}$ \\
\hline Age (years) & 1.014 & $0.981-1.048$ & 0.409 \\
Sex (male) & 0.730 & $0.361-1.477$ & 0.381 \\
Presence of hypertension & 0.819 & $0.254-2.638$ & 0.737 \\
Presence of diabetes mellitus & 0.300 & $0.069-1.307$ & 0.109 \\
Presence of hyperlipidemia & 0.351 & $0.172-0.717$ & 0.004 \\
Presence of smoking & 1.854 & $0.553-6.214$ & 0.317 \\
Neutrophil to lymphocyte ratio & 2.451 & $1.423-4.222$ & 0.001 \\
\hline
\end{tabular}

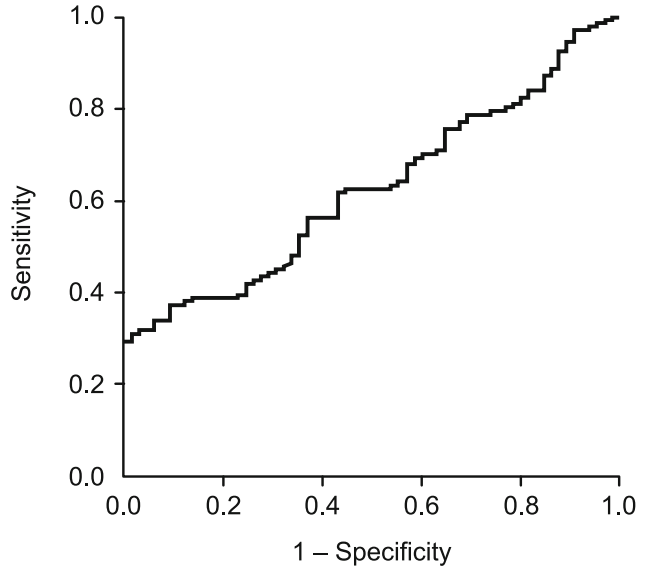

Fig. 3. Receiver operating characteristic (ROC) curve analysis for neutrophil to lymphocyte ratio to predict obstructive sleep apnea (area under curve is $\mathbf{0 . 6 2 3}$ ).

5-15, 16-30, > 30, respectively). Patients with higher AHI score had significantly higher NLR (normal: $1.49 \pm 0.48$, mild OSA: 1.64 \pm 0.65 , moderate OSA: $1.88 \pm 0.79$, severe OSA: $1.92 \pm 0.83, \mathrm{p}=$ $0.002)$. Correlation analysis revealed that NLR was significantly correlated with AHI score $(r=0.228, p=0.001)$ (Fig. 2). There was no significant correlation between CRP levels and AHI score in patients with OSA $(r=0.157, p=0.084)$. Logistic regression analysis revealed that NLR and presence of HL were independent predictors of OSA(OR: $2.451,95 \% \mathrm{CI}$ : $1.423-4.222, \mathrm{p}=0.001$ and OR: $2.850,95 \%$ CI: $1.395-5.824, \mathrm{p}=0.004$, respectively) (Tab. 3 ). ROC analysis was performed to determine the cut-off level of NLR to predict the OSA. A NLR of 1.62 or higher predicted OSA with a sensitivity of $56.2 \%$ and specificity of $63.1 \%$ (Fig. 3).

\section{Discussion}

In this study, we evaluated the relationship between NLR and OSA. Our results demonstrated that NLR was significantly higher and also correlated with AHI scores in patients with OSA. Moreover, NLR was an independent predictor of OSA which has not been reported previously.

Sleep abnormalities are associated with poor cardiovascular outcomes $(14,15)$. OSA is an important health care problem which is also linked to cardiovascular risk factors including HT, DM, HL, obesity, coronary artery disease, and stroke (16). Approximately half of patients with OSA have HT. There are numerous mechanisms linking OSA and HT such as excessive sympathetic activity, increased heart rate and peripheral vascular resistance (17). In our study, presence of HT and heart rate were significantly higher in patients with OSA consistent with previous study. Additionally, DM and HL were also more common in patients with OSA in accordance with previous studies (18).

There is a closer relationship between OSA and obesity. Previous studies have demonstrated that the incidence of obesity is approximately $60-90 \%$ in patients with OSA (19). In our study, patients with OSA had significantly higher BMI levels compared 
to controls $(34.4 \pm 7.8$ vs $26.9 \pm 4.9, \mathrm{p}<0.001)$. Numerous studies have demonstrated that inflammation is the main factor for the relationship between OSA and CVD. Inflammation plays an important role in the pathogenesis of atherosclerosis (20). Hypoxia and systemic inflammation may lead to progressive atherosclerosis and contribute to increased risk of CVD in patients with OSA (21-23). Increased levels of inflammatory markers are associated with increased cardiovascular risk (24). Patients with OSA have higher levels of inflammatory markers such as CRP, neutrophil counts, TNF- $\alpha$, pro-inflammatory cytokines, interleukin (IL)-6, serum amyloid A, and leukocyte adhesion molecules (5, 6, 25-27). Therefore, it is not surprising that OSA patients have increased risk of CVD (28).

The neutrophil to lymphocyte ratio has been proposed as a new biomarker for predicting CVD (10). Neutrophils and leukocytes are the most common types of white blood cells and they play an important role in the development of inflammatory response. They release several types of cytokines and trigger the immune system and inflammatory reactions. Previous studies have demonstrated that higher levels of NLR are associated with impaired cardiovascular outcomes (10-13). NLR is not only associated with CVD, but also associated with chronic renal disease and several malignancies $(11,13,29,30)$. Although numerous studies have investigated the relationship between NLR and CVD, the relationship between NLR and OSA remains unclear. NLR was relatively lower in our study population when compared to previous studies with different population characteristics. In a previous study, Tamhane et al (10) investigated the association between admission NLR and outcomes in patients with ACS. NLR was 1.82 in low tertile, 3.56 in medium tertile, and 9.10 in high tertile in their study population. The study population was older than our study population (61-65 years versus 49 years, respectively). On the other hand, while our study population consisted of OSA patients with no systemic disease and ACS, their study population consisted of patients with ACS. Therefore, increased tissue damage due to acute myocardial injury may contribute to higher NLR as well as older study population. In another study, Bhatti et al (30) demonstrated that the preoperative NLR is a significant independent prognostic indicator in patients with resected pancreatic ductal adenocarcinoma. The median age was 65 years and median NLR was 2.4 in their study population. The study population consisted of patients with pancreatic ductal adenocarcinoma. In addition to older study population, promoting cancer cell proliferation, ongoing angiogenesis, tumor metastasis and effect of systemic therapies may also contribute to higher NLR compared to our results. In our study, we first demonstrated that NLR was higher and also significantly correlated with AHI scores in patients with OSA. Moreover, NLR was an independent predictor for OSA. Although CRP levels were also higher in patients with OSA, it was not a predictor for OSA in our study. NLR is also inexpensive, routinely used, reproducible test that has been used as a biomarker to evaluate inflammatory state in outpatient clinical setting. Therefore, simple and cheap complete blood counts may also give an idea about the severity of OSA in outpatient clinics.

Our study had several limitations. Our study had a cross-sectional design, it would be better to follow patients with OSA and look for the risk factors in the ones who will develop CVD after a certain period of time. On the other hand, we did not evaluate the effect of treatment on NLR in patients with OSA. It would be better to follow patients after adequate time following treatment. For this purpose, further prospective studies are needed to determine the effect of treatment on NLR and the prognosis of these patients.

In conclusion, NLR was higher and also correlated with AHI score in patients with OSA which has not been reported previously. Moreover, NLR more than 1.62 was an independent predictor of OSA. A simple, cheap white blood cell count may also give an idea about the presence and severity of OSA and it may be useful in outpatient clinical setting.

\section{References}

1. Qureshi A, Ballard RD. Obstructive sleep apnea. J Allergy Clin Immunol 2003; 112 (4): 643-651.

2. Young T, Palta M, Dempsey J, Skatrud J, Weber S, Badr S. The occurrence of sleep disordered breathing among middle aged adults. N Engl J Med 1993; 328: 1230-1235.

3. Parish JM, Somers VK. Obstructive sleep apnea and cardiovascular disease. Mayo Clin Proc 2004; 79 (8): 1036-1046.

4. Williams A, Scharf SM. Obstructive sleep apnea, cardiovascular disease, and inflammation--is NF-kappaB the key? Sleep Breath 2007; 11 (2): 69-76.

5. Dyugovskaya L, Lavie P, Lavie L. Increased adhesion molecules expression and production of reactive oxygen species in leukocytes of sleep apnea patients. Am J Respir Crit Care Med 2002; 165: 859-860.

6. Larkin EK, Rosen CL, Kirchner HL et al. Variation of C-reactive protein levels in adolescents: association with sleep-disordered breathing and sleep duration. Circulation 2005; 111: 1978-1984.

7. Ryan S, Taylor CT, McNicholas WT. Selective activation of inflammatory pathways by intermittent hypoxia in obstructive sleep apnea syndrome. Circulation 2005; 112: 2660-2667.

8. Lavie L. Obstructive sleep apnoea syndrome-an oxidative stress disorder. Sleep Med Rev 2003; 7: 35-51.

9. Ayas NT, Mancini GB, Fleetham J. Does CPAP delay the development of cardiovascular disease in patients with obstructive sleep apnoea hypopnoea? Thorax 2006; 61: 459-460.

10. Tamhane UU, Aneja S, Montgomery D, Rogers EK, Eagle KA, Gurm HS. Association between admission neutrophil to lymphocyte ratio and outcomes in patients with acute coronary syndrome. Am J Cardiol 2008; 102 (6): 653-657.

11. Okyay GU, Inal S, Oneç K et al. Neutrophil to lymphocyte ratio in evaluation of inflammation in patients with chronic kidney disease. Ren Fail 2013; 35 (1): 29-36.

12. Gibson PH, Cuthbertson BH, Croal BL et al. Usefulness of neutrophil/lymphocyte ratio as predictor of new-onset atrial fibrillation after coronary artery bypass grafting. Am J Cardiol 2010; 105: 186-191.

13. Sunbul M, Gerin F, Durmus E et al. Neutrophil to lymphocyte and platelet to lymphocyte ratio in patients with dipper versus non-dipper hypertension. Clin Exp Hypertens 2014; 36 (4): 217-221.

14. Wolk R, Gami AS, Garcia-Touchard A, Somers VK. Sleep and cardiovascular disease. Curr Probl Cardiol 2005; 30: 625-662. 


\section{4-658}

15. Sunbul M, Kanar BG, Durmus E, Kivrak T, Sari I. Acute sleep deprivation is associated with increased arterial stiffness in healthy young adults. Sleep Breath 2014; 18 (1): 215-220.

16. Lattimore JD, Celermajer DS, Wilcox I. Obstructive sleep apnea and cardiovascular disease. J AmColl Cardiol 2003; 41: 1429-1437.

17. Stradling J, Davies RJ. Sleep apnea and hypertension - what a mess! Sleep 1997; 20: 789-793.

18. Pamidi S, Tasali E. Obstructive sleep apnea and type 2 diabetes: is there a link? Front Neurol 2012; 3:126.

19. Young T, Peppard PE, Gottlieb DJ. Epidemiology of obstructive sleep apnea: a population health perspective. Am J Respir Crit Care Med 2002; 165: 1217-1239.

20. Badran M, Ayas N, Laher I. Insights into obstructive sleep apnea research. Sleep Med 2014; 15 (5): 485-495.

21. Guven SF, Turkkani MH, Ciftci B, Ciftci TU, Erdogan Y. The relationship between high-sensitivity C-reactive protein levels and the severity of obstructive sleep apnea. Sleep Breath 2012; 16 (1): 217-221.

22. Williams A, Scharf SM. Obstructive sleep apnea, cardiovascular disease, and inflammation - is NF-kappaB the key? Sleep Breath 2007; 11 (2): 69-76.

23. Altekin RE, Yanikoğlu A, Karakaş MS, Ozel D, Yildirim AB, Kabukçu M. Evaluation of subclinical left ventricular systolic dysfunction in patients with obstructive sleep apnea by automated function imaging method; an observational study. Anadolu Kardiyol Derg 2012; 12 (4): 320-330.
24. Libby P, Willerson JT, Braunwald E. C-reactive protein and coronary heart disease. N Engl J Med 2004; 351: 295-298.

25. Yokoe T, Minoguchi K, Matsuo H et al. Elevated levels of C-reactive protein and interleukin-6 in patients with obstructive sleep apnea syndrome are decreased by nasal continuous positive airway pressure. Circulation 2003; 107: 1129-1134.

26. Karakaş MS, Altekin RE, Baktir AO, Küçük M, Cilli A, Yalçinkaya $\mathbf{S}$. Association between mean platelet volume and severity of disease in patients with obstructive sleep apnea syndrome without risk factors for cardiovascular disease. Turk Kardiyol Dern Ars 2013; 41 (1): 14-20.

27. Bravo Mde L, Serpero LD, Barceló A, Barbé F, Agustí A, Gozal D. Inflammatory proteins in patients with obstructive sleep apnea with and without daytime sleepiness. Sleep Breath 2007; 11 (3): 177-185.

28. Shah NA1, Yaggi HK, Concato J, Mohsenin V. Obstructive sleep apnea as a risk factor for coronary events or cardiovascular death. Sleep Breath 2010; 14 (2): 131-136.

29. Halazun KJ, Aldoori A, Malik HZ et al. Elevated preoperative neutrophil to lymphocyte ratio predicts survival following hepatic resection for colorectal liver metastases. Eur J Surg Oncol 2008; 34: 55-60.

30. Bhatti I, Peacock O, Lloyd G, Larvin M, Hall RI. Preoperative hematologic markers as independent predictors of prognosis in resected pancreatic ductal adenocarcinoma: neutrophil-lymphocyte versus plateletlymphocyte ratio. Am J Surg 2010; 200: 197-203.

Received December 9, 2014. Accepted June 26, 2015. 